\title{
EVALUATION OF TWO CONVOLUTION SUMS INVOLVING THE SUM OF DIVISORS FUNCTION
}

\section{Mathieu Lemire and Kenneth S. Williams}

The convolution sums $\sum_{m<n / 5} \sigma(m) \sigma(n-5 m)$ and $\sum_{m<n / 7} \sigma(m) \sigma(n-7 m)$ are evaluated for all $n \in \mathbb{N}$.

\section{INTRODUCTION.}

Let $\mathbb{N}$ denote the set of natural numbers. We set

$$
\sigma_{k}(n)=\sum_{d \mid n} d^{k}, \quad n, k \in \mathbb{N}
$$

where $d$ runs through the positive integers dividing $n$. If $n \notin \mathbb{N}$ we set $\sigma_{k}(n)=0$. We write $\sigma(n)$ for $\sigma_{1}(n)$. We define the convolution sum $W_{k}(n)$ by

$$
W_{k}(n):=\sum_{m<n / k} \sigma(m) \sigma(n-k m)
$$

where $m$ runs through the positive integers $<n / k$. The sum $W_{k}(n)$ has been evaluated for $k=1,2,3,4,6,8,9$ and 16 for all $n \in \mathbb{N}$, see $[4,7](k=1),[7,8,9](k=2,3,4)$, $[2](k=6),[11](k=8),[8,9,10,12](k=9),[1](k=16)$. For $k=5$ Melfi $[8,9]$ has shown that for $n \equiv 8(\bmod 16), n \not \equiv 0(\bmod 5)$

$$
W_{5}(n)=\frac{5}{312} \sigma_{3}(n)+\left(\frac{1}{24}-\frac{1}{20} n\right) \sigma(n) .
$$

In this paper we make use of some recent results of Berndt, Chan, Sohn and Son $[\mathbf{3}]$ to evaluate $W_{5}(n)$ and $W_{7}(n)$ for all $n \in \mathbb{N}$. We prove

THEOREM 1. For all $n \in \mathbb{N}$

$$
W_{5}(n)=\frac{5}{312} \sigma_{3}(n)+\frac{125}{312} \sigma_{3}\left(\frac{n}{5}\right)+\left(\frac{1}{24}-\frac{1}{20} n\right) \sigma(n)+\left(\frac{1}{24}-\frac{1}{4} n\right) \sigma\left(\frac{n}{5}\right)-\frac{1}{130} c_{5}(n),
$$

where the $c_{5}(n)(n \in \mathbb{N})$ are integers defined by

$$
q \prod_{n=1}^{\infty}\left(1-q^{n}\right)^{4}\left(1-q^{5 n}\right)^{4}=\sum_{n=1}^{\infty} c_{5}(n) q^{n}
$$

Received 5th October, 2005

Research of the second author was supported by Natural Sciences and Engineering Research Council of Canada grant A-7233.

Copyright Clearance Centre, Inc. Serial-fee code: 0004-9727/06 \$A2.00+0.00. 
The first twenty values of $c_{5}(n)$ are $c_{5}(1)=1, c_{5}(2)=-4, c_{5}(3)=2, c_{5}(4)=8$, $c_{5}(5)=-5, c_{5}(6)=-8, c_{5}(7)=6, c_{5}(8)=0, c_{5}(9)=-23, c_{5}(10)=20, c_{5}(11)=32$, $c_{5}(12)=16, c_{5}(13)=-38, c_{5}(14)=-24, c_{5}(15)=-10, c_{5}(16)=-64, c_{5}(17)=26$, $c_{5}(18)=92, c_{5}(19)=100, c_{5}(20)=-40$.

THEOREM 2. For all $n \in \mathbb{N}$

$$
W_{7}(n)=\frac{1}{120} \sigma_{3}(n)+\frac{49}{120} \sigma_{3}\left(\frac{n}{7}\right)+\left(\frac{1}{24}-\frac{1}{28} n\right) \sigma(n)+\left(\frac{1}{24}-\frac{1}{4} n\right) \sigma\left(\frac{n}{7}\right)-\frac{1}{70} c_{7}(n),
$$

where the $c_{7}(n)(n \in \mathbb{N})$ are integers defined by

$$
\begin{aligned}
q\left(\prod_{n=1}^{\infty}\left(1-q^{n}\right)^{16}\left(1-q^{7 n}\right)^{8}+13 q\right. & \prod_{n=1}^{\infty}\left(1-q^{n}\right)^{12}\left(1-q^{7 n}\right)^{12} \\
& \left.+49 q^{2} \prod_{n=1}^{\infty}\left(1-q^{n}\right)^{8}\left(1-q^{7 n}\right)^{16}\right)^{1 / 3}=\sum_{n=1}^{\infty} c_{7}(n) q^{n} .
\end{aligned}
$$

The first twenty values of $c_{7}(n)$ are $c_{7}(1)=1, c_{7}(2)=-1, c_{7}(3)=-2, c_{7}(4)=-7$, $c_{7}(5)=16, c_{7}(6)=2, c_{7}(7)=-7, c_{7}(8)=15, c_{7}(9)=-23, c_{7}(10)=-16, c_{7}(11)=-8$, $c_{7}(12)=14, c_{7}(13)=28, c_{7}(14)=7, c_{7}(15)=-32, c_{7}(16)=41, c_{7}(17)=54, c_{7}(18)=23$, $c_{7}(19)=-110, c_{7}(20)=-112$.

\section{NOTATION.}

Let $z=x+i y \in \mathbb{C}$ be such that $y>0$. Set $q=e^{2 \pi i z}$ so that $|q|=e^{-2 \pi y}<1$. The Dedekind eta function $\eta(z)$ is defined by

$$
\eta(z):=e^{2 \pi i z / 24} \prod_{n=1}^{\infty}\left(1-e^{2 \pi i n z}\right) .
$$

Following Ramanujan (see [3, p. 82]) we set

$$
f(-q):=e^{-2 \pi i z / 24} \eta(z)=\prod_{n=1}^{\infty}\left(1-e^{2 \pi i n z}\right)=\prod_{n=1}^{\infty}\left(1-q^{n}\right) .
$$

It is convenient to set

$$
A:=f(-q), B:=f\left(-q^{5}\right), C:=f\left(-q^{7}\right) .
$$

We define $c_{5}(n)$ and $c_{7}(n)$ by (1.3) and (1.4) respectively. It is clear that $c_{5}(n) \in \mathbb{Z}$. We shall show that $c_{7}(n) \in \mathbb{Z}$ in the proof of Theorem 2. From (1.3), (1.4), (2.2) and (2.3), we obtain

$$
\begin{aligned}
q A^{4} B^{4} & =\sum_{n=1}^{\infty} c_{5}(n) q^{n} . \\
q A^{3} B^{3}\left(\frac{A^{7}}{C}+13 q A^{3} C^{3}+49 q^{2} \frac{C^{7}}{A}\right)^{1 / 3} & =\sum_{n=1}^{\infty} c_{7}(n) q^{n} .
\end{aligned}
$$


The Eisenstein series $P(q)$ and $Q(q)$ (see [3, p. 81]) are defined by

$$
P(q):=1-24 \sum_{n=1}^{\infty} \sigma(n) q^{n}
$$

and

$$
Q(q):=1+240 \sum_{n=1}^{\infty} \sigma_{3}(n) q^{n}
$$

\section{Proof of Theorem 1.}

We begin with a classical result.

LEMMa 3.1.

$$
P(q)^{2}=1+\sum_{n=1}^{\infty}\left(240 \sigma_{3}(n)-288 n \sigma(n)\right) q^{n}
$$

Proof: See for example Glaisher $[5,6]$.

LEMMA 3.2 .

$$
P\left(q^{5}\right)^{2}=1+\sum_{n=1}^{\infty}\left(240 \sigma_{3}\left(\frac{n}{5}\right)-\frac{288}{5} n \sigma\left(\frac{n}{5}\right)\right) q^{n} .
$$

Proof: Letting $q \rightarrow q^{5}$ in Lemma 3.1 we obtain the result.

LEMMA 3.3 .

$$
\left(P(q)-5 P\left(q^{5}\right)\right)^{2}=16 \frac{A^{10}}{B^{2}}+352 q A^{4} B^{4}+2000 q^{2} \frac{B^{10}}{A^{2}} .
$$

Proof: This follows from [3, (3.21), (3.34)] and (2.3).

LEMMA 3.4 .
(a)
$Q(q)=\frac{A^{10}}{B^{2}}+250 q A^{4} B^{4}+3125 q^{2} \frac{B^{10}}{A^{2}}$.
(b)

$$
Q\left(q^{5}\right)=\frac{A^{10}}{B^{2}}+10 q A^{4} B^{4}+5 q^{2} \frac{B^{10}}{A^{2}}
$$

Proof: This follows from [3, Theorem 3.1, p. 85] and (2.3).

LEMMA 3.5 .

$$
\begin{aligned}
\text { (a) } \frac{A^{10}}{B^{2}} & =1+\sum_{n=1}^{\infty}\left(-\frac{5}{13} \sigma_{3}(n)+\frac{3125}{13} \sigma_{3}\left(\frac{n}{5}\right)-\frac{125}{13} c_{5}(n)\right) q^{n} . \\
\text { (b) } q^{2} \frac{B^{10}}{A^{2}} & =\sum_{n=1}^{\infty}\left(\frac{1}{13} \sigma_{3}(n)-\frac{1}{13} \sigma_{3}\left(\frac{n}{5}\right)-\frac{1}{13} c_{5}(n)\right) q^{n} .
\end{aligned}
$$


Proof: From Lemma 3.4 we obtain

$$
\frac{A^{10}}{B^{2}}=-\frac{1}{624} Q(q)+\frac{625}{624} Q\left(q^{5}\right)-\frac{125}{13} q A^{4} B^{4}
$$

and

$$
q^{2} \frac{B^{10}}{A^{2}}=\frac{1}{3120} Q(q)-\frac{1}{3120} Q\left(q^{5}\right)-\frac{1}{13} q A^{4} B^{4} .
$$

Letting $q \rightarrow q^{5}$ in (2.7), we obtain

$$
Q\left(q^{5}\right)=1+240 \sum_{n=1}^{\infty} \sigma_{3}\left(\frac{n}{5}\right) q^{n}
$$

Using (2.4), (2.7) and (3.3) in (3.1) and (3.2), we obtain the asserted results.

LEMMA 3.6 .

$$
\left(P(q)-5 P\left(q^{5}\right)\right)^{2}=16+\sum_{n=1}^{\infty}\left(\frac{1920}{13} \sigma_{3}(n)+\frac{4800}{13} \sigma_{3}\left(\frac{n}{5}\right)+\frac{576}{13} c_{5}(n)\right) q^{n} .
$$

PRoof: This follows from Lemma 3.3, Lemma 3.5 and (2.4).

\section{LEMMA 3.7 .}

$$
P(q) P\left(q^{5}\right)=1+\sum_{n=1}^{\infty}\left(\frac{120}{13} \sigma_{3}(n)+\frac{3000}{13} \sigma_{3}\left(\frac{n}{5}\right)-\frac{144}{5} n \sigma(n)-144 n \sigma\left(\frac{n}{5}\right)-\frac{288}{65} c_{5}(n)\right) q^{n}
$$

Proof: This follows from Lemmas 3.1, 3.2, 3.6 and the identity

$$
P(q) P\left(q^{5}\right)=\frac{1}{10} P(q)^{2}+\frac{5}{2} P\left(q^{5}\right)^{2}-\frac{1}{10}\left(P(q)-5 P\left(q^{5}\right)\right)^{2} .
$$

Proof of Theorem 1. Letting $q \rightarrow q^{5}$ in (2.6), we obtain

$$
P\left(q^{5}\right)=1-24 \sum_{n=1}^{\infty} \sigma\left(\frac{n}{5}\right) q^{n}
$$

Next

$$
\begin{aligned}
\sum_{n=1}^{\infty} W_{5}(n) q^{n} & =\sum_{n=1}^{\infty}\left(\sum_{m<n / 5} \sigma(m) \sigma(n-5 m)\right) q^{n} \\
& =\left(\sum_{l=1}^{\infty} \sigma(l) q^{l}\right)\left(\sum_{m=1}^{\infty} \sigma(m) q^{5 m}\right) \\
& =\left(\frac{1-P(q)}{24}\right)\left(\frac{1-P\left(q^{5}\right)}{24}\right) \\
& =\frac{1}{576}-\frac{1}{576} P(q)-\frac{1}{576} P\left(q^{5}\right)+\frac{1}{576} P(q) P\left(q^{5}\right)
\end{aligned}
$$


Appealing to (2.6), (3.1) and Lemma 3.7, and equating coefficients of $q^{n}$ ( $n \in \mathbb{N}$ ), we obtain the required result.

Since $c_{5}(m n)=c_{5}(m) c_{5}(n)$ for all $m, n \in \mathbb{N}$ with $(m, n)=1$ and $c_{5}(8)=0$ (see [9]), we deduce that $c_{5}(n)=0$ for all $n \in \mathbb{N}$ with $n \equiv 8(\bmod 16)$. Hence for $n \equiv 8(\bmod 16)$ Theorem 1 gives

$$
W_{5}(n)=\frac{5}{312} \sigma_{3}(n)+\frac{125}{312} \sigma_{3}\left(\frac{n}{5}\right)+\left(\frac{1}{24}-\frac{1}{20} n\right) \sigma(n)+\left(\frac{1}{24}-\frac{1}{4} n\right) \sigma\left(\frac{n}{5}\right) .
$$

Melfi's result (1.2) is the special case of this result when $n \not \equiv 0(\bmod 5)$.

We show that the evaluation

$$
c_{5}\left(5^{k}\right)=(-1)^{k} 5^{k}, \quad k \in \mathbb{N} \cup\{0\}
$$

is a consequence of Theorem 1 . Let $n \in \mathbb{N}$. The elementary identities

$$
\sigma(5 n)=6 \sigma(n)-5 \sigma(n / 5)
$$

and

$$
\sigma_{3}(5 n)=126 \sigma_{3}(n)-125 \sigma(n / 5)
$$

are easily proved. Thus

$$
\begin{aligned}
W_{5}(5 n) & =\sum_{m<n} \sigma(m) \sigma(5 n-5 m) \\
& =6 \sum_{m<n} \sigma(m) \sigma(n-m)-5 \sum_{m<n} \sigma(m) \sigma((n-m) / 5)
\end{aligned}
$$

It is a classical result that

$$
\sum_{m<n} \sigma(m) \sigma(n-m)=\frac{5}{12} \sigma_{3}(n)+\left(\frac{1}{12}-\frac{n}{2}\right) \sigma(n),
$$

see for example [7]. Also

$$
\sum_{m<n} \sigma(m) \sigma((n-m) / 5)=\sum_{t<n / 5} \sigma(n-5 t) \sigma(t)=W_{5}(n)
$$

Hence

$$
W_{5}(5 n)+5 W_{5}(n)=\frac{5}{2} \sigma_{3}(n)+\left(\frac{1}{2}-3 n\right) \sigma(n) .
$$

Appealing to Theorem 1, (3.6) and (3.7), we obtain

$$
c_{5}(5 n)=-5 c_{5}(n), \quad n \in \mathbb{N}
$$

from which (3.5) follows. 


\section{Proof of Theorem 2.}

It is convenient to set

$$
H:=\left(\frac{A^{7}}{C}+13 q A^{3} C^{3}+49 q^{2} \frac{C^{7}}{A}\right)^{1 / 3},
$$

where $A$ and $C$ are defined in (2.3).

LEMMA 4.1.

$$
P\left(q^{7}\right)^{2}=1+\sum_{n=1}^{\infty}\left(240 \sigma_{3}\left(\frac{n}{7}\right)-\frac{288}{7} n \sigma\left(\frac{n}{7}\right)\right) q^{n}
$$

Proof: Letting $q \rightarrow q^{7}$ in Lemma 3.1, we obtain the result.

LEMMA 4.2 .

$$
\left(P(q)-7 P\left(q^{7}\right)\right)^{2}=36 H^{4} .
$$

Proof: From [3, Lemma 6.2, p. 109] we obtain

$$
P(q)-7 P\left(q^{7}\right)=-6\left(\frac{A^{7}}{C}\right)^{2 / 3}\left(1+13 q \frac{C^{4}}{A^{4}}+49 q^{2} \frac{C^{8}}{A^{8}}\right)^{2 / 3}
$$

Squaring both sides we obtain

$$
\begin{aligned}
\left(P(q)-7 P\left(q^{7}\right)\right)^{2} & =36\left(\frac{A^{7}}{C}\right)^{4 / 3}\left(1+13 q \frac{C^{4}}{A^{4}}+49 q^{2} \frac{C^{8}}{A^{8}}\right)^{4 / 3} \\
& =36\left(\frac{A^{7}}{C}+13 q A^{3} C^{3}+49 q^{2} \frac{C^{7}}{A}\right)^{43} \\
& =36 H^{4}
\end{aligned}
$$

LEMMA 4.3 .

(a)

$$
\begin{array}{ll}
\text { (a) } & Q(q)=\left(\frac{A^{7}}{C}+245 q A^{3} B^{3}+2401 q^{2} \frac{C^{7}}{A}\right) H . \\
\text { (b) } & Q\left(q^{7}\right)=\left(\frac{A^{7}}{C}+5 q A^{3} B^{3}+q^{2} \frac{C^{7}}{A}\right) H .
\end{array}
$$

Proof: This follows from [3, Theorem 5.1, p. 100].

LEMMA 4.4 .

$$
q A^{3} B^{3} H=\sum_{n=1}^{\infty} c_{7}(n) q^{n}
$$

Proof: This follows from (2.5) and (4.1).

LEMMA 4.5 .

(a) $\frac{A^{7}}{C} H=1+\sum_{n=1}^{\infty}\left(-\frac{1}{10} \sigma_{3}(n)+\frac{2401}{10} \sigma_{3}\left(\frac{n}{7}\right)-\frac{49}{10} c_{7}(n)\right) q^{n}$.

(b) $\quad q^{2} \frac{C^{7}}{A} H=\sum_{n=1}^{\infty}\left(\frac{1}{10} \sigma_{3}(n)-\frac{1}{10} \sigma_{3}\left(\frac{n}{7}\right)-\frac{1}{10} c_{7}(n)\right) q^{n}$. 
ProOF: From Lemma 4.3 we obtain

$$
\frac{A^{7}}{C}=-\frac{1}{2400} Q(q)+\frac{2401}{2400} Q\left(q^{7}\right)-\frac{49}{10} q A^{3} C^{3} H
$$

and

$$
q^{2} \frac{C^{7}}{A} H=\frac{1}{2400} Q(q)-\frac{1}{2400} Q\left(q^{7}\right)-\frac{1}{10} q A^{3} C^{3} H .
$$

Letting $q \rightarrow q^{7}$ in (2.7) we obtain

$$
Q\left(q^{7}\right)=1+240 \sum_{n=1}^{\infty} \sigma_{3}\left(\frac{n}{7}\right) q^{n} .
$$

Using (2.7), (4.4) and Lemma 4.3 in (4.2) and (4.3), we obtain the asserted resuits.

LEMMA 4.6 .

$$
\left(P(q)-7 P\left(q^{7}\right)\right)^{2}=36+\sum_{n=1}^{\infty}\left(\frac{864}{5} \sigma_{3}(n)+\frac{42336}{5} \sigma_{3}\left(\frac{n}{7}\right)+\frac{576}{5} c_{7}(n)\right) q^{n} .
$$

Proof: This follows from Lemmas 4.2, 4.5 and 4.6.

LEMMA 4.7.

$$
\begin{aligned}
P(q) P\left(q^{7}\right)=1+\sum_{n=1}^{\infty}\left(\frac{24}{5} \sigma_{3}(n)+\frac{1176}{5} \sigma_{3}\left(\frac{n}{7}\right)-\frac{144}{7} n \sigma(n)\right. & \\
& \left.\quad-144 n \sigma\left(\frac{n}{5}\right)-\frac{288}{35} c_{7}(n)\right) q^{n} .
\end{aligned}
$$

Proof: This follows from Lemmas 3.1, 4.1, 4.7 and the identity

$$
P(q) P\left(q^{7}\right)=\frac{1}{14} P(q)^{2}+\frac{49}{14} P\left(q^{7}\right)^{2}-\frac{1}{14}\left(P(q)-7 P\left(q^{7}\right)\right)^{2} .
$$

Proof OF TheOREM 2. Letting $q \rightarrow q^{7}$ in (2.6), we obtain

$$
P\left(q^{7}\right)=1-24 \sum_{n=1}^{\infty} \sigma\left(\frac{n}{7}\right) q^{n}
$$

Next

$$
\begin{aligned}
\sum_{n=1}^{\infty} W_{7}(n) q^{n} & =\sum_{n=1}^{\infty}\left(\sum_{m<\frac{n}{7}} \sigma(m) \sigma(n-7 m)\right) q^{n} \\
& =\left(\sum_{l=1}^{\infty} \sigma(l) q^{l}\right)\left(\sum_{m=1}^{\infty} \sigma(m) q^{7 m}\right) \\
& =\left(\frac{1-P(q)}{24}\right)\left(\frac{1-P\left(q^{7}\right)}{24}\right) \\
& =\frac{1}{576}-\frac{1}{576} P(q)-\frac{1}{576} P\left(\dot{q}^{7}\right)+\frac{1}{576} P(q) P\left(q^{7}\right)
\end{aligned}
$$


Appealing to (2.6), (4.5) and Lemma 4.7, and equating coefficients of $q^{n}$, we obtain the formula for $W_{7}(n)$ in Theorem 2.

We now show that the $c_{7}(n)$ are integers. It is easy to check that for all $n \in \mathbb{N}$ we have

$$
\sigma_{3}(n) \equiv \sigma(n)(\bmod 3)
$$

and

$$
\sigma_{3}(n) \equiv(2 n-1) \sigma(n)(\bmod 4)
$$

Hence

$$
\begin{aligned}
& 7 \sigma_{3}(n)+343 \sigma_{3}(n / 7)+(35-30 n) \sigma(n)+(35-210 n) \sigma(n / 7) \\
& \equiv \sigma_{3}(n)+\sigma_{3}(n / 7)-\sigma(n)-\sigma(n / 7) \equiv 0(\bmod 3)
\end{aligned}
$$

and

$$
\begin{aligned}
7 \sigma_{3}(n)+343 \sigma_{3}(n / 7)+(35-30 n) \sigma(n)+(35-210 n) \sigma(n / 7) \\
\equiv-\sigma_{3}(n)-\sigma_{3}(n / 7)-(1+2 n) \sigma(n)-(1+2 n) \sigma(n / 7) \equiv 0(\bmod 4)
\end{aligned}
$$

so

$$
7 \sigma_{3}(n)+343 \sigma_{3}(n / 7)+(35-30 n) \sigma(n)+(35-210 n) \sigma(n / 7) \equiv 0(\bmod 12) .
$$

Thus

$$
e(n):=\frac{7 \sigma_{3}(n)+343 \sigma_{3}(n / 7)+(35-30 n) \sigma(n)+(35-210 n) \sigma(n / 7)}{12} \in \mathbb{Z} .
$$

Hence

$$
c_{7}(n)=e(n)-70 W_{7}(n) \in \mathbb{Z} .
$$

Similarly to the proof of (3.5) we can use Theorem 2 to show that

$$
c_{7}\left(7^{k}\right)=(-1)^{k} 7^{k}, \quad k \in \mathbb{N} \cup\{0\}
$$

\section{REFERENCES}

[1] A. Alaca, S. Alaca and K.S. Williams, 'The convolution sum $\sum_{m<n / 16} \sigma(m) \sigma(n-16 m)$ ',

[2] S. Alaca and K.S. Williams, 'The convolution sums $\sum_{l+6 m=n} \sigma(l) \sigma(m)$ and $\sum_{2 l+3 m=n} \sigma(l) \sigma(m)^{\prime}$, (submitted).

[3] B.C. Berndt, H.H. Chan, J. Sohn and S.H. Son, 'Eisenstein series in Ramanujan's lost notebook', Ramanujan J. 4 (2000), 81-114.

[4] M. Besge, 'Extrait d'une lettre de M. Besge à M. Liouville', J. Math. Pures Appl. 7 (1862), 256.

[5] J.W.L. Glaisher, 'On the square of the series in which the coefficients are the sums of the divisors of the exponents', Mess. Math. 14 (1885), 156-163. 
[6] J.W.L. Glaisher, Mathematical Papers 1883-1885 (Cambridge, 1885).

[7] J.G. Huard, Z.M. Ou, B.K. Spearman and K.S. Williams, 'Elementary evaluation of certain convolution sums involving divisor functions', in Number Theory for the Millenium II (A.K. Peters, Natick, MA, 2002), pp. 229-274.

[8] G. Melf, Some problems in elementary number theory and modular forms, Ph.D thesis (University of Pisa, 1998).

[9] G. Melf, 'On some modular identities', in Number Theory (de Gruyter, Berlin, 1998), pp. 371-382.

[10] K.S. Williams, 'A cubic transformation formula for ${ }_{2} F_{1}\left(\frac{1}{3}, \frac{2}{3} ; 1, z\right)$ and some aritímeicic convolution formulae', Math. Proc. Cambridge Philos. Soc. 137 (2004), 519-539.

[11] K.S. Williams, 'The convolution sum $\sum_{m<n / 8} \sigma(m) \sigma(n-8 m)$ ', Pacific J. Math. (to appear). [12] K.S. Williams, 'The convolution sum $\sum_{m<n / 9} \sigma(m) \sigma(n-9 m)$ ', Int. J. Number Theory 1
(2005), 193-205.

School of Mathematics and Statistics

Carleton University

Ottawa, Ontario K1S 5B6

Canada

e-mail: mathieul@math.carleton.ca
Centre for Research in Algebra and Number Theory

School of Mathematics and Statistics

Carleton University

Ottawa, Ontario K1S 5B6

Canada

e-mail: williams@math.carleton.ca 